\title{
Intracochlearendoscopy in Cochlear Implantation of X-Linked Stapes Gusher Syndrome
}

\author{
Jennifer $\mathrm{Ha}^{1,2}$, Timothy Baerg ${ }^{2}$ and Marc C Thorne ${ }^{2}$
}

${ }^{1}$ Department of Paediatrics Otolaryngology Head \& Neck Surgery, Perth Children's Hospital, 15 Hospital Avenue, Nedlands, 6009, Western Australia, Australia; School of Surgery, University of Western Australia, Stirling Highway, Crawley, Perth 6009, Western Australia; Murdoch ENT, Wexford Medical Center, Suite 17-18, Level 1, 3 Barry Marshall Parade, Murdoch 6150, Western Australia

${ }^{2}$ Department of Otolaryngology Head and Neck Surgery, University of Michigan Medical School, USA

\begin{abstract}
Introduction: X-linked stapes gusher syndrome is a rare form of congenital sensorineural hearing loss (SNHL). Affected patients have abnormal configuration of the lamina cribrosa and internal auditory canal (IAC), leading to an increased risk of perilymph gusher with surgical manipulation. We report a case whereby intra-operative too endoscopic visualization allowed real time visualization of the inner ear (IE) anatomy, which has the potential to impact on electrode choice in cochlear implants $(\mathrm{Cl})$.

Case: A 12-year-old boy has been followed up in our Pediatrics Otolaryngology department with bilateral SNHL and X-linked stapes gusher syndrome for five years. He initially performed well with bilateral traditional hearing aids, but developed progressive mixed loss and became a $\mathrm{Cl}$ candidate for his left ear. The pre-operative computed tomography of the temporal bone showed the characteristic bulbous IAC with a dysplastic cochlea and no apparent modiolus. Intraoperative intracochlear endoscopy however, showed an apparent modiolus without direct communication to the IAC, contrary to the imaging.
\end{abstract}

Discussion: Electrode choice during $\mathrm{Cl}$ in patients with IE malformation is based on the expected location of spiral ganglion cells. Intracochlear endoscopy offers an additional tool for assessment of inner ear anatomy, which may assist in optimal electrode selection.

Conclusion: We advocate the use of intracochlear endoscopy in selected cases as it offers a better assessment of the intracochlear membranous compartments and modiolusthan high resolution CT and has the potential to allow customization of electrode choice based on membranous inner ear anatomy.

\section{Keywords}

Cochlear Endoscopy, Otoendoscopy, X-Linked Stapes Gusher Syndrome, Cochlear Implant, Per Lymphatic Gusher

\section{Introduction}

X-linked stapes gusher syndrome (otherwise known as $\mathrm{X}$-linked deafness type 3 or Nance deafness) is a rare form of congenital mixed hearing loss. Inherited in a sex-linked recessive manner, it is believed to be the consequence of a lossof-function mutation on the X-chromosome in the POU3F4 gene at the DFN3 locus [1]. Males tend to demonstrate more severe phenotypic presentations than females do, who present uncommonly and with less severity [2].

Affected patients have conductive hearing loss from stapes fixation and often have progressive sensorineural hearing loss (SNHL). These patients have an abnormal configuration of the lamina cribrosa and internal auditory canal (IAC), which results in an increased risk of cerebrospinal fluid (CSF) gusher with surgical manipulation [2-4]. This may lead to other complications, such as, CSF rhinorrhea and recurrent meningitis

\footnotetext{
*Corresponding author: Jennifer Ha, Department of Otolaryngology - Head \& Neck Surgery, Division of Pediatric Otolaryngology, CS Mott Children's Hospital, University of Michigan Health System, 1540 E Hospital Drive, SPC 4241, Ann Arbor, Michigan 48109-4241, USA, phone: +1 734936 4934, Fax: +1 734763 7802

Accepted: November 28, 2020

Published online: November 30, 2020

Citation: Ha J, Baerg T, Thorne MC (2020) Intracochlearendoscopy in Cochlear Implantation of X-Linked Stapes Gusher Syndrome. Arch of Pedia Surg 4(2):72-74
} 


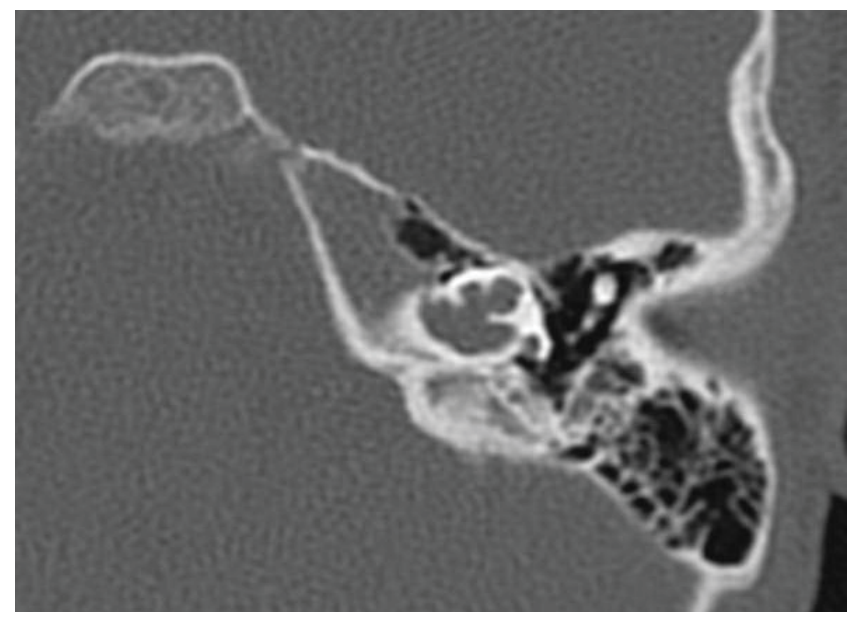

Figure 1: Pre-operative audiogram with speech testing

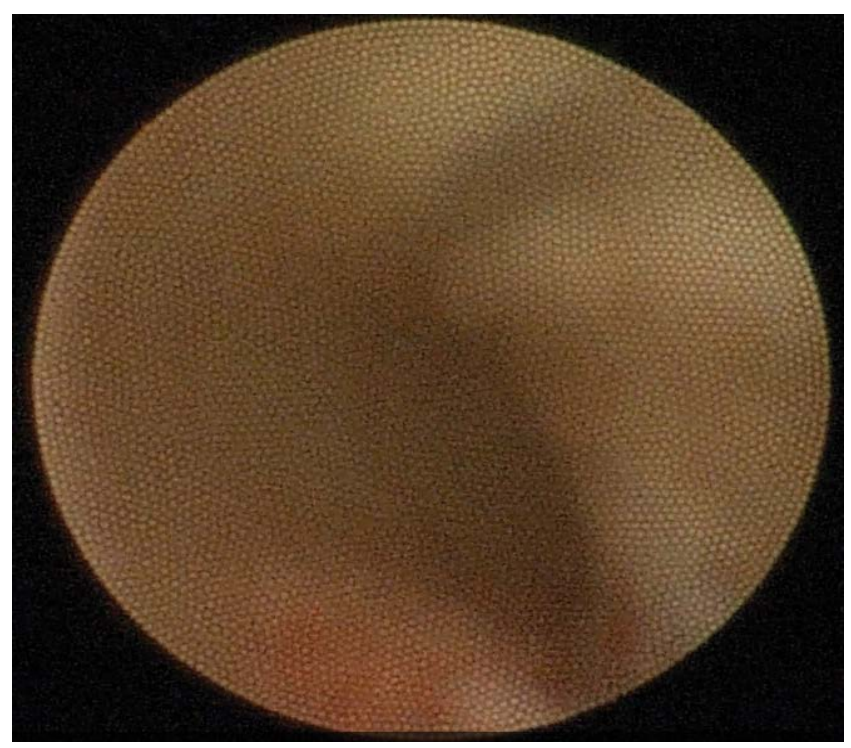

Figure 2: Dysplastic cochlear with no modiolus on imaging

[2]. Meningitis complicating cochlear implantation (Cl) occurs at a higher rate in patients with inner ear (IE) abnormalities, including $\mathrm{X}$-linked gusher syndrome $[2,5]$.

Traditional approaches to imaging for $\mathrm{Cl}$ utilize computed tomography of the temporal bone (CTTB) and /or magnetic resonance imaging. We report a case whereby intra-operative cochlear intra endoscopic visualization allowed real time visualization of the IE anatomy, which has the potential to impact on electrode choices in $\mathrm{Cl}$. Visualization of the membranous portions of the IE might allow for selection of an electrode best able to stimulate existing neural elements.

\section{Case Report}

A 12-year-old boy with X-linked stapes gusher syndrome initially performed well with bilateral traditional hearing aids, but developed progressive mixed loss and worsening performance of his left ear. His pre-operative audiogram is shown in (Figure 1).

The pre-operative CTTB showed a bulbous IAC with a dysplastic cochlea and no apparent modiolus characteristic of
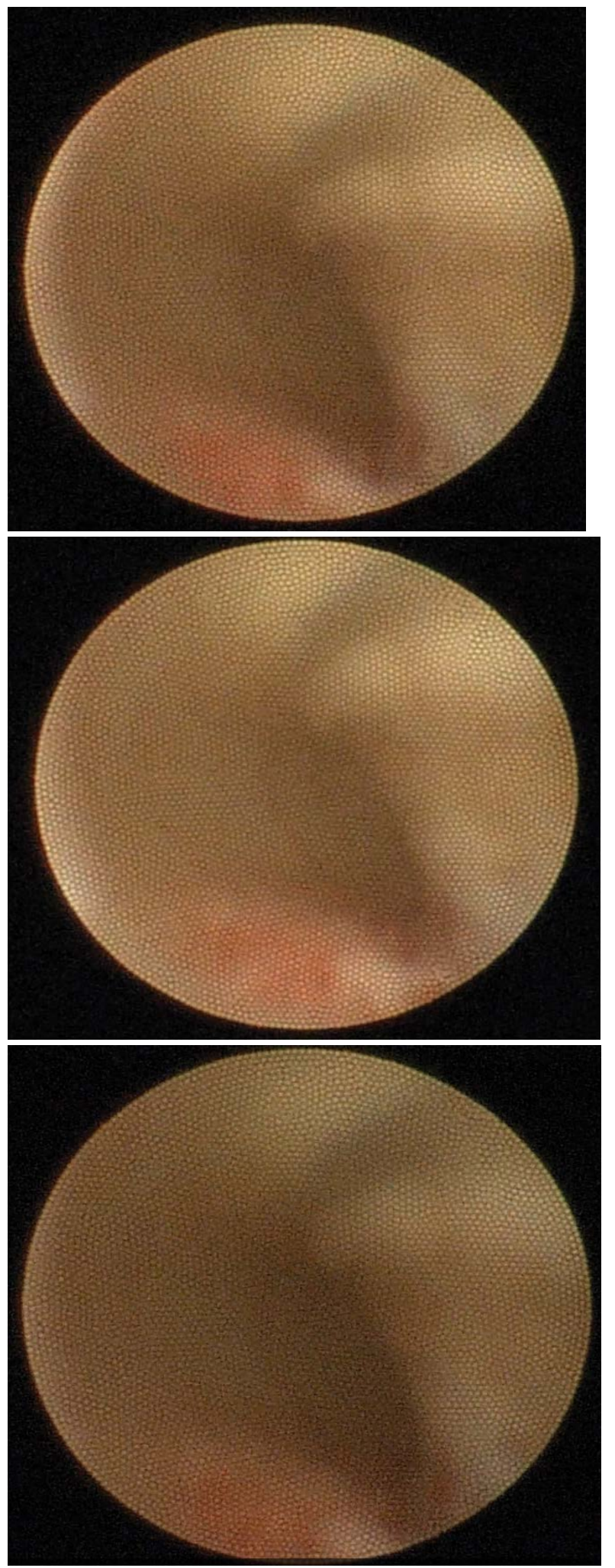

Figure 3: Cochlear endoscopy showing membranous structure with no obvious connection to the IAC.

this condition (Figure 2). A CI24RE (ST) implant was selected by the $\mathrm{Cl}$ team due to uncertainty regarding the location of the spiral ganglion cells.

The standard approach was utilized for his $\mathrm{Cl}$. A $1 \mathrm{~mm}$ cochleostomy was made anteroinferior to the round window 
(RW). Upon reaching the cochleostomy, a moderate CSF gusher was encountered. A $1.3 \mathrm{~mm}$ salivary endoscope was placed at the entrance of the cochlea to specifically assess whether there was an absent modiolus. Upon visualization, there was a modiolus without direct communication to the IAC (Figure 3). Full insertion was achieved on the first attempt without any difficulty. The gusher was controlled with packing of periosteal tissue at the cochleostomy site. An intra-operative plain X-ray confirmed the electrode placement.

\section{Discussion}

When the cochlear modiolus and osseous spiral lamina are deficient, this absent bony septum creates a common space seen on the imaging study (Figure 2) [6]. This abnormal communication between the IAC and vestibule is responsible for the CSF gusher. Patients with IE anomalies may have both fewer and atypical positions of their spiral ganglion cells (SGC) [7]. This has functional implications because at least 10000 functional SGCs are necessary for effective speech discrimination [8]. This number can be further reduced by surgical trauma [8]. When there is uncertainty regarding the likely location of spiral ganglion cells, a fully banded $\mathrm{Cl}$ electrode may be chosen to allow for full and multi-directional stimulation, whereas a directional electrode may be more appropriate if there is greater confidence in the location of spiral ganglion cells for precise stimulation [9].

The proper electrode choice may have important hearing outcome implications for patients with IE anomalies following $\mathrm{Cl}$ due to challenges of reaching an optimal level of cochlear stimulation, decreased dynamic range, a wider pulse width, and weakened neural synchrony [9]. The functionality of $\mathrm{Cl}$ is correlated to the number of SGCs and their distances from the stimulating electrode [10]. While CTTB provides important information for surgical planning, the resolution is not currently sufficient to assess the fine detail of the structures of the inner ear. Our case demonstrates that intra-operative intra endoscopic findings may identify anatomic features not available on $\mathrm{CT}$, indicating that cochlear endoscopy may be a useful tool to better assess the intracochlear anatomy. The intra endoscopic intracochlear view gave us accurate and real-time information on the anatomy of the IE, which confirmed the presence of a modiolus. Although we did not alter electrode choice in this patient, the endoscopic findings suggest that a directional electrode may have been appropriate.

This assumes that SGCs are likely to be centrally located when membranous inner ear structures are preserved, as opposed to a peripheral location seen in common cavity malformations [6]. We suggest thatcochlear intraendoscopy allows better delineation of the intracochlear anatomy, which may impact the electrode selection and potentially the hearing outcomes in patients with anomalous inner ear anatomy orambiguous locations of spiral ganglion cells. Potential risks to cochlearintraendoscopy are insertion trauma or damage to delicate auditory and vestibular structures.

\section{Conclusion}

We advocate the use of intracochlear endoscopy in selected cases as it offers a better assessment of intracochlear membranous compartments and modiolusthan the high resolution CTTB. This has the potential to allow customization of electrode choice based on membranous inner ear anatomy, and potentially the hearing outcomes in patients with anomalous IE anatomy and uncertain location of spiral ganglion cells.

\section{Acknowledgements}

None.

\section{Financial Support/Funding}

None to declare.

\section{Conflict of Interest}

None to declare.

\section{References}

1. Huang BY, Zdanski C, Castillo M, et al. (2011) Pediatric sensorineural hearing loss, part 2: Syndromic and acquired causes. American Journal of Neuroradiolog 33: 399-406.

2. Incesulu A, Adapinar B, Kecik C, et al. (2008) Cochlear implantation in cases with incomplete partition type iii (x-linked anomaly). European Archives of Oto-Rhino-Laryngology 265: 1425-1430.

3. Kumar G, Castillo M, Buchman CA, et al. (2003) X-linked stapes gusher: $\mathrm{Ct}$ findings in one patient. American journal of neuroradiology 24: 1130-1132.

4. Pakdaman MN, Herrmann BS, Curtin HD, et al. (2012) Cochlear implantation in children with anomalous cochleovestibular anatomy: A systematic review. Otolaryngology -head neck surg 146:180-190.

5. Cohen NL, Roland JT, Marrinan M, et al. (2004) Meningitis in cochlear implant recipients:The north american experience. Otology \& Neurotology 25: 275-281.

6. Nager GT, Hyams VJ (1993) Pathophysiology of the ear and temporal bone. Baltimore: Lippincott williams \& wilkins, December.

7. Busi M, Rosignoli M, Castiglione A, et al. (2015) Cochlear implant outcomes and genetic mutations in children with ear and brain anomalies. BioMed Research International, 1-19.

8. Otte J, Schuknecht HF, Kerr AG, et al. (1978) Ganglion cell populations in normal and pathological human cochleae. Implications for cochlear implantation. The Laryngoscope 88: 1231-1246.

9. Hughes ML, Abbas PJ (2006) Electrophysiologic channel interaction, electrode pitch ranking, and behavioral threshold in straight versus perimodiolar cochlear implant electrode arrays. The Journal of the Acoustic Society of America 119: 1538-1547.

10. Khan AM, Levine SR, Nadol JB, et al. (2006) The widely patent cochleovestibular communication of edward cock is a distinct inner ear malformation: Implications for cochlear implantation. Annals of Otology, Rhinology \& Laryngology 115: 595-606.

DOI: $10.36959 / 472 / 358$

Copyright: (C) 2020 Jennifer $\mathrm{FH}$, et al. This is an open-access article distributed under the terms of the Creative Commons Attribution License, which permits unrestricted use, distribution, and reproduction in any medium, provided the original author and source are credited. 